\title{
A contact cavity-biased method for grand canonical Monte Carlo simulations
}

\author{
Danny H. L. Yau, Steven Y. Liem, and Kwong-Yu Chan ${ }^{\text {a) }}$ \\ Department of Chemistry, The University of Hong Kong, Pokfulam Road, Hong Kong
}

(Received 18 May 1994; accepted 26 July 1994)

\begin{abstract}
A modification of the cavity-biased grand canonical Monte Carlo (GCMC) proposed by Mezei is introduced here. Instead of on a fixed grid, test points of cavities are generated at the contact positions around the centers of existing particles. The increase in the probability or bias of finding a cavity is related to the radial distribution function and can hence be corrected. With this new cavity-biased implementation, an improved convergence to equilibrium is demonstrated and higher densities can be attained. Comparisons with the standard GCMC method, and the original cavity-biased scheme of Mezei are made.
\end{abstract}

\section{INTRODUCTION}

The grand canonical Monte Carlo (GCMC) method developed over the past two decades ${ }^{1-3}$ has been popular in the studies of fluids, in particular, for mixtures of fluids and phase equilibrium studies. In this method, the chemical potential is fixed and the system density is allowed to fluctuate with insertion and deletion of particles. Equilibrium is attained when the number of successful insertion and deletion attempts balance each other. Application of this technique at high density states, however, is difficult because the probability of finding a large enough cavity for particle insertion is small. Mezei ${ }^{4}$ proposed that this difficulty can be overcome by a biased-sampling method in which insertions are made at locations where cavities exist. The bias is corrected by reducing the insertion acceptance with a factor corresponding to the probability of finding a cavity. This probability of finding a cavity is determined by testing a number of locations in each Monte Carlo move and at the same time the cavities in each move are located. At moderate densities, this cavity bias scheme shows a marked improvement over the standard GCMC method ${ }^{5}$ and has been applied to several different systems. ${ }^{6,7}$ At high densities, say at reduced density $\rho^{*}$ larger than 0.6 , the probability of finding a cavity is small and a large number of test points and a large amount of cpu time are required for the cavity-biased GCMC method. In the original scheme of cavity biased GCMC proposed by Mezei, a fixed grid of test points is used throughout the simulation. At high densities, if the test points all overlap with the particle coordinates, it will take many MC moves before a cavity exists when the particles slowly translate away through the canonical MC moves. It is therefore advantageous to modify Mezei's method by avoiding the centers of the particles. By generating test points at a distance radially outward from the centers of the particles, we find an increased likelihood of finding a cavity. Furthermore, the test points will change with each MC move and can minimize the situation with a deadlock configuration. The probability of finding a cavity as a function of the distance from the center of a particle correlates with the radial distribution function, according to the scaled-particle theory. ${ }^{8}$ Hence the radial bias

a) Author to whom correspondence should be addressed. can be estimated and corrected. The maximum likelihood of finding a cavity, as with the maximum of the pair radial distribution function, occurs at the distance $\sigma$, the diameter of the particle. A contact-cavity biased GCMC method in which test points are at a distance $\sigma$ away from the centers of existing particles, will have the highest efficiency. In this paper, we will discuss the motivation and some preliminary implementations of this contact cavity-biased GCMC method. Simulations of the hard sphere fluid at a wide range of chemical potentials are reported. Comparisons with the standard GCMC method; the original Mezei's cavity biased method, and the Carnahan-Starling equation ${ }^{9}$ will be made wherever possible.

\section{CAVITY-BIASED GCMC}

\section{A. Grand canonical Monte Carlo simulation}

In a GCMC simulation, the chemical potential $(\mu)$, temperature $(T)$, and volume $(V)$ are fixed and the number of particles or the density is allow to fluctuate. The grand canonical ensemble partition function is

$$
\exists=\sum_{N=0}^{\infty} \exp \left(\frac{N \mu}{k_{B} T}\right) \int_{V} \exp \left[-\frac{U\left(r^{N}\right)}{k_{B} T}\right] d r^{N} /\left(\Lambda^{3 N} N !\right),
$$

where $N$ is the number of particles, $r^{N}$ is the coordinate vector in the configuration space of $N$ particles, $V$ is the volume of the system, $U\left(r^{N}\right)$ is the potential energy of the configuration $r^{N}, k_{B}$ is the Boltzmann factor, $T$ is absolute temperature, and $\Lambda$ is the thermal deBroglie wavelength given by

$$
\Lambda=\frac{h}{\sqrt{2 \pi m k_{B} T}} .
$$

In a normal GCMC simulation, there are three types of moves; (i) a displacement move to displace a particle from its original position; (ii) a random deletion move where a particle is randomly selected and destroyed; and (iii) a random insertion move where a particle is created at a random position in the system. From Eq. (1), a random insertion attempt is accepted with the probability 


$$
\begin{aligned}
P_{i}= & \min \left(1, V \exp \left\{\left[\mu+U\left(r^{N}\right)\right.\right.\right. \\
& \left.\left.\left.-U\left(r^{N+1}\right)\right] / k_{B} T\right\} / \Lambda^{3}(N+1)\right)
\end{aligned}
$$

and a random deletion attempt is accepted with probability

$$
\begin{aligned}
P_{d}= & \min \left(1, N \exp \left\{\left[-\mu+U\left(r^{N}\right)\right.\right.\right. \\
& \left.\left.\left.-U\left(r^{N-1}\right)\right] / k_{B} T\right\} \Lambda^{3} / V\right),
\end{aligned}
$$

where $N$ is the number of particles before the insertion/ deletion attempt. Even though GCMC offers a significant advantage over simulation in the canonical ensemble, the efficiency of this approach decreases with the increase of the system's density. At high densities, insertion attempts are rejected because of the high occurrence of overlapping configurations. For hard core and repulsive potentials, this problem is more pronounced and there are many rejections of insertion attempts at high densities. For a hard sphere fluid, this difficulty is encountered at $\rho^{*}>0.6$.

\section{B. Cavity-biased GCMC}

A modified GCMC method which can be used to study systems at higher density was proposed by Mezei in the early 1980 s. He suggested a modification of the insertion process which allows for insertion only at points where a cavity of suitable radius exists $\left(R_{c} \geqslant \sigma / 2\right)$. In this case the acceptance probabilities for insertion and deletion, $P_{i}$ and $P_{d}$ will be modified as the following:

$$
\begin{aligned}
P_{i}^{C B}= & \min \left(1, V P_{c} \exp \left\{\left[\mu+U\left(r^{N}\right)\right.\right.\right. \\
& \left.\left.\left.-U\left(r^{N+1}\right)\right] / k_{B} T\right\} / \Lambda^{3}(N+1)\right), \\
P_{d}^{C B}= & \min \left(1, N \exp \left\{\left[-\mu+U\left(r^{N}\right)\right.\right.\right. \\
& \left.\left.\left.-U\left(r^{N-1}\right)\right] / k_{B} T\right\} \Lambda^{3} /\left(V P_{c}\right)\right),
\end{aligned}
$$

where $P_{c}$ is the probability of finding a cavity of radius $R_{c}$ or larger in a system of $N$ particles. The realization of the proposed sampling thus requires the computation of $P_{c}$ and the finding of a point that is the center of a cavity of radius $R_{c}$ or larger. By generating a number of uniformly distributed test points, and finding the fraction of those are in a suitable cavity, one obtains both an estimate for $P_{c}$ and also a definite point where a new particle can be inserted. As the simulation proceeds, the estimate of $P_{c}$ will necessarily improve. In the event where no cavity exists at any of the test points, the standard GCMC method will be applied where a random deletion or insertion is attempted with the GCMC acceptance probability in Eqs. (2) or (3).

The cavity-biased GCMC method yields a high acceptance ratio of insertion and deletion of particles and can sample higher density states. The success of this method depends on finding at least one cavity in the system and therefore a larger Ntest will be needed at higher densities.

According to the Widom's equation, ${ }^{10,11}$ the chemical potential of a test particle in a system of $N$ particles is

$$
\mu=k_{B} T \ln \left(\rho_{N-1} \Lambda^{3}\right)-k_{B} T \ln \left\langle\exp \left(-\Delta U_{\text {test }} / k_{B} T\right)\right\rangle
$$

therefore,

$$
\mu^{\mathrm{ex}}=-k_{B} T \ln \left\langle\exp \left(-\Delta U_{\text {test }} / k_{B} T\right)\right\rangle,
$$

where $\Delta U_{\text {test }}$ is the potential energy change which would result from the addition of a particle at random to the system. Considering the term $\exp \left(-\Delta U_{\text {test }} / k_{B} T\right)$ in a hard sphere syslem, $\Delta U_{\text {test }}$ is equal to zero when there is no overlap or infinity when there is an overlap. Therefore the term $\exp \left(-\Delta U_{\text {test }} / k_{B} T\right)$ is equal to 1 or 0 . Accordingly, in the cavity biased method with Ntest test points,

$$
\begin{aligned}
& \mu^{\mathrm{ex}}=-k_{B} T \ln \left(N_{\mathrm{cav}} / \text { Ntest }\right), \\
& \mu^{\mathrm{ex}}=-k_{B} T \ln P_{c},
\end{aligned}
$$

where $P_{c}$ is the probability of finding a cavity and $N_{\text {cav }}$ is the number of cavities found. This result is the same as reported in de Souza et al. ${ }^{12}$ The excess chemical potential is related to the density by the Carnahan-Starling equation

$$
\mu^{\mathrm{ex}}=k T\left[-3+(3-\eta) /(1-\eta)^{3}\right] .
$$

The above equation is derived by integrating the pressure to obtain the free energy, $A$. The pressure, according to the Carnahan and Starling expression, ${ }^{9}$ is

$$
P V / N k T=\left(1+\eta+\eta^{2}-\eta^{3}\right)(1-\eta)^{-3},
$$

where $\eta$ is the packing fraction, $\eta=(\pi / 6) \rho \sigma^{3}$. Therefore the probability of finding a cavity, as a function of density is

$$
\ln \left(P_{c}\right)=3-(3-\eta) /(1-\eta)^{3} \text {. }
$$

At a high density, say $\rho^{*}=0.8, P_{c}$ is $O\left(10^{-5}\right)$ which means that a minimum value of Ntest $=100000$ is needed before a cavity can be found. This places a heavy demland of cpu time.

\section{MODIFIED CAVITY-BIASED GCMC}

\section{A. Radial correlation of cavities}

In order to study hard sphere fluids at high reduced densities, a modification of the cavity-biased GCMC method is investigated in this paper. In this modification, the test points are not uniformly distributed within the system and change as the simulation proceeds. Each test point is generated at a distance away from a randomly selected particle in the system. This distance of insertion can be chosen by convenience. The probability of finding a cavity, $P_{c}$ depends on the distance from the central particle where the insertion is attempted. According to the scaled particle theory, ${ }^{8}$ the radial distribution of the probability of finding a cavity, given a hard sphere in the center, is just the hard sphere pair distribution function,

$$
P_{c}(r) / P_{c}(\infty)=g(r),
$$

where $P_{c}(\infty)$ is the probability of finding a cavity at infinite distance from the central hard particle. The highest $P_{c}$, is therefore, at the contact position of each particle.

To compare the cavity probabilities at various density states, canonical (NVT) MC runs are made with 1000 hard spheres. For each simulation, the probability of finding a cavity is estimated using Mezei's method as well as the radial sampling method. The value of $P_{c}$ according to Mezei's method is calculated on an evenly spaced $10 \times 10 \times 10$ lattice points. This value of $P_{c}$ is equivalent to $P_{c}(\infty)$ in the radial sampling scheme. $P_{c}(r)$ in the radial cavity search scheme is 
TABLE I. Probabilities of finding a cavity, $P_{e}$ at $1.00001 \sigma$ and $5 \sigma$ away from a particle and that on a fixed grid.

\begin{tabular}{|c|c|c|c|c|c|}
\hline \multirow[b]{2}{*}{$\rho^{*}$} & \multicolumn{3}{|c|}{$P_{\bar{c}}$} & \multirow[b]{2}{*}{ (b) $/(\mathrm{c})$} & \multirow[b]{2}{*}{$g(1.00000 \sigma$} \\
\hline & $\begin{array}{c}\text { (a) } \\
\text { Radial } \\
\text { sampling }(5 \sigma)\end{array}$ & $\begin{array}{c}\text { (b) } \\
\text { Contact } \\
(1.00001 \sigma)\end{array}$ & $\begin{array}{c}\text { (c) } \\
\text { Mezei's } \\
\text { method }\end{array}$ & & \\
\hline 0.2 & 0.358895 & 0.461706 & 0.357751 & 1.29058 & 1.29207 \\
\hline 0.3 & 0.177419 & 0.266069 & 0.176566 & 1.50691 & 1.51315 \\
\hline 0.4 & $0.733781 \times 10^{-1}$ & 0.128344 & $0.728188 \times 10^{-1}$ & 1.762 .51 & 1.76939 \\
\hline 0.5 & $0.22541 \times 10^{-1}$ & $0.483685 \times 10^{-1}$ & $0.225118 \times 10^{-1}$ & 2.14858 & 2.13382 \\
\hline 0.6 & $0.507189 \times 10^{-2}$ & $0.133763 \times 10^{-1}$ & $0.504416 \times 10^{-2}$ & 2.65184 & 2.48156 \\
\hline 0.7 & $0.6379 \times 10^{-3}$ & $0.21819 \times 10^{-2}$ & $0.594905 \times 10^{-3}$ & 3.66764 & 3.37772 \\
\hline 0.8 & $0.4480 \times 10^{-4}$ & $0.15160 \times 10^{-3}$ & $0.323855 \times 10^{-4}$ & 4.68111 & 4.56350 \\
\hline 0.9 & $0.1200 \times 10^{-5}$ & $0.5375 \times 10^{-5}$ & $0.100 \times 10^{-5}$ & $5.3750^{\circ}$ & 5.54164 \\
\hline
\end{tabular}

evaluated by generating one test point at a fixed distance $r$, but at a randomly chosen angle, around each particle with a total of 1000 test points for each insertion attempt. The cavity probabilities near contact $P_{c}(1.00001 \sigma)$, the $P_{c}$ at $5 \sigma$, and the $P_{c}$ on a grid (Mezei's method) are compared in Table I. The results are averaged over 10 million $\mathrm{MC}$ moves after equilibration. Except at high densities $\left(\rho^{*}>0.7\right)$, the $P_{c}$ at $5 \sigma$ and the $P_{c}$ on a grid (Mezei's method) agree within $1 \%$ equivalent. Figure 1 shows the comparison of these probabilities as well as the prediction according to CarnahanStarling equation and the Widom's equation as in Eq. (11). The discrepancies at high densities may be due to poor statistics of insufficient cavities but it seems that in general, the radial cavity probability at long range is larger than that on a grid. The results of Table I shows that the increase in probability near contact is directly proportional to the distribution function. At $p^{*}-0.9$, it is 5 times more likely to find a cavity near contact. To test Eq. (12) and the radial dependence of the likelihood of a cavity, $P_{c}(r)$, more canonical $(N V T)$ MC simulations were made for 256 hard spheres at two reduced densities $\rho^{*}=0.5$ and 0.7 . Four test points at a distance $r$ around each particle are used with a total of 1024 test points for each insertion attempt. The $P_{c}$ at various radial distances

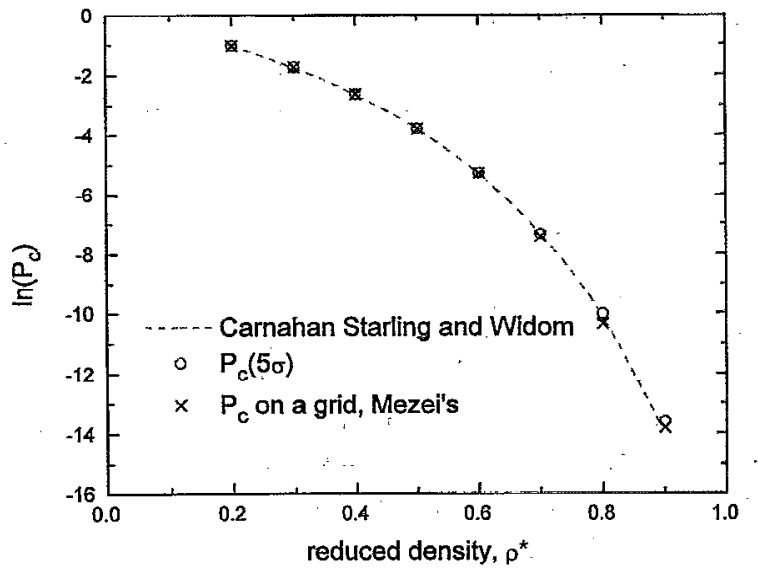

FIG. 1. Probabilities of finding a cavity, $P_{\mathrm{c}}$ according to Carnahan-Starling and Widom's equation, radial cavity biased GCMC (at $5 \sigma$ ) and Mezei's cavity biased GCMC. are shown in Figs. 2 and 3 for the low and high densities, respectively. It is shown that Eq. (12) is accurate in predicting the radial dependence of $P_{c}$.

\section{B. Frequency correction factor}

Comparing the cavity probabilities in Table I, attempting insertion near contact is easier to succeed in GCMC. The same acceptance probabilities of Eqs. (4) and (5) can be used. In using these equations, the value of $P_{c}$ should be the noncontact biased value and can be obtained by Eq. (12) since both $P_{c}(\sigma)$ and $g(\sigma)$ are available in the simulation. Theoretically, if the value of $P_{c}(\infty)$ is known, e.g., by Eq. (11), there is no need to find more than one cavity in each insertion attempt and the more efficient way of finding a cavity, e.g., the contact-cavity biased scheme, the better. This will be true if there is more than one cavity in each insertion attempt. At high densities, however, $P_{c}(\infty)$ can be as low as $10^{-6}$ and it will not be possible to find a cavity in each insertion move. The contact-insertion scheme will then have a bias over the long range insertion scheme when the former method finds a cavity more often than the latter. To correct for this bias, the probability of finding at least one cavity,

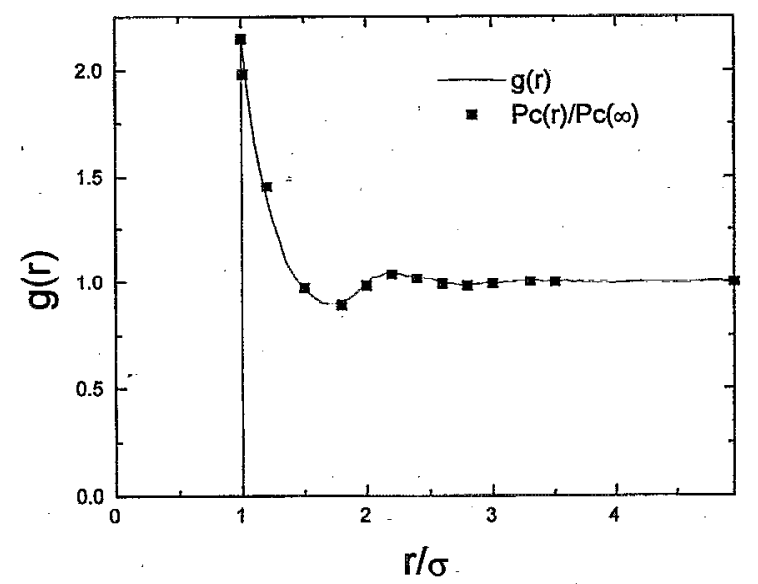

FIG. 2. Comparison of radial distribution function, $g(r)$ with $P_{c}(r) / P_{c}(\infty)$ at 0.5 reduced density. 


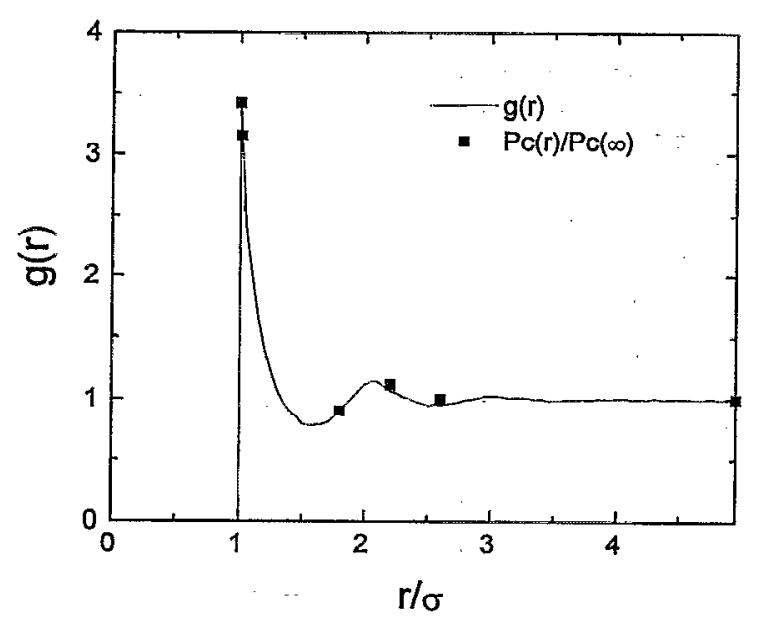

FIG. 3. Comparison of radial distribution function, $g(r)$ with $P_{c}(r) / P_{c}(\infty)$ at 0.7 reduced density.

$P_{c 1^{+}}(r)$ which depends on the number of test points, is calculated. This is equal to one minus the probability of not finding any cavity in Ntest trials,

$$
P_{c 1+}(r)=1-\left[1-P_{c}(r)\right]^{\text {Ntest }} \text {. }
$$

This bias of finding at least one cavity more often can be corrected by introducing a frequency correction factor in the acceptance probability expressions of Eqs. (4) and (5),

$$
\begin{aligned}
P_{i}^{C B}= & \min \left(1, V f_{c} P_{c} \exp \left\{\left[\mu+U\left(r^{N}\right),\right.\right.\right. \\
& \left.\left.\left.-U\left(r^{N+1}\right)\right] / k_{B} T\right\} / \Lambda^{3}(N+1)\right), \\
P_{d}^{C B}= & \min \left(1, N \exp \left\{\left[-\mu+U\left(r^{N}\right)\right.\right.\right. \\
& \left.\left.\left.-U\left(r^{N-1}\right)\right] / k_{B} T\right\} \Lambda^{3} /\left(V f_{c} P_{c}\right)\right),
\end{aligned}
$$

where $f_{c}$ is equal to the ratio of the probabilities of finding at least one cavity,

$$
f_{c}=P_{c 1+}(\infty) / P_{c 1+}(\sigma) .
$$

At low densities or high $P_{c}$, or Ntest is large, $f_{c}$ is close to unity. At high densities, this factor is less than one and if omitted, will lead to errors in the final density.

From the results of some test runs, the dependence of the frequency correction factor on density and Ntest is illustrated in Figs. 4 and 5, respectively. The individual $P_{c 1+}$ at $1.01 \sigma$ is compared with that of Mezei's method at different densities. At low densities, the two probabilities are identical and the frequency correction factor is close to one. At densities above 0.6, the frequency correction factor is much below unity. In Fig. 5, increase in the number of test points will help to narrow the difference of insertion near contact and insertion at $4.0 \sigma$.

\section{Test of the algorithm}

The contact-biased GCMC algorithm is implemented with the acceptance probability of Eqs. (14) and (15). Comparisons are made with Mezei's method of insertion on a regular grid. To compare the two methods on the same basis, insertions and deletions are made only at the end of a cycle,

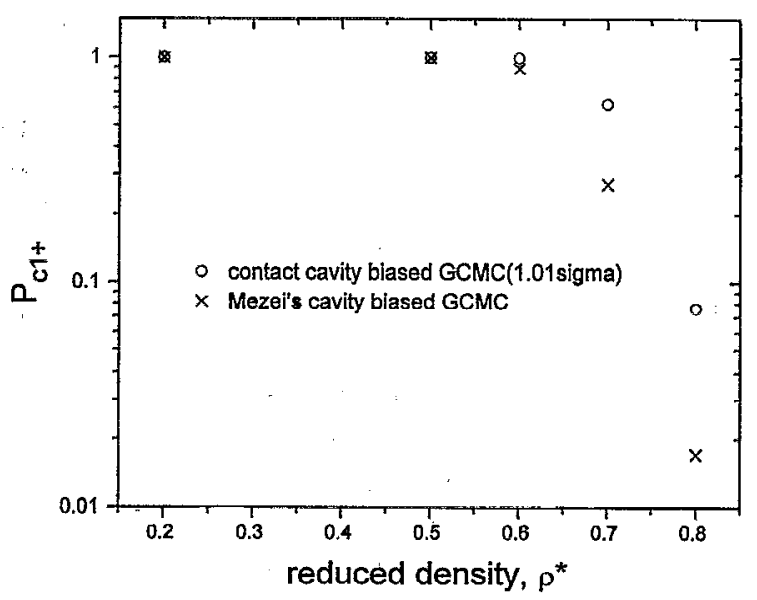

FIG. 4. Comparison of the probabilities of finding at least one cavity, $P_{c 1+}$ between contact cavity biased GCMC (1.01 sigma) and Mezei's cavity biased GCMC method.

i.e., when attempted displacements of all the particles have been made. The starting initial configuration is at $\rho^{*}=0.2$ with 256 particles. The total number of cavity test points is 1000. This corresponds to ten grids on each side of the simulation box in Mezei's method or 1 to 4 test points per particle in the new method. At a reduced activity of 100 and 100000 , both algorithms converge to the same final density after the same number of insertion attempts. From Figs. 6 and 7, the contact insertion algorithm tends to have a larger overshoot in number of particles before converging to the final number. It appears that contact cavity bias is not more efficient and requires the same number of insertion moves. However, one additional advantage of the contact cavity bias is that insertion attempts can be made many times in a MC cycle before every particle is displaced. In cavity sampling on a fixed grid, there is not much change in the sampling until most of the particles are displaced. Accordingly, more insertion attempts can be made with the new algorithm. Subsequently, extra runs are made with more insertions per cycle for the

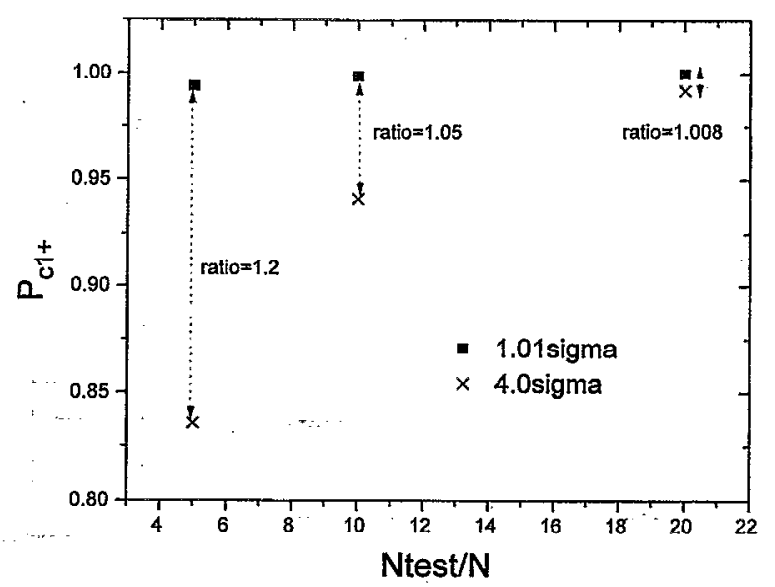

FIG. 5. The dependence of the frequency correction factor on the number of cavity test points per particle, Ntest $/ N$ sampling at $1.01 \sigma$ and $4.0 \sigma$. 


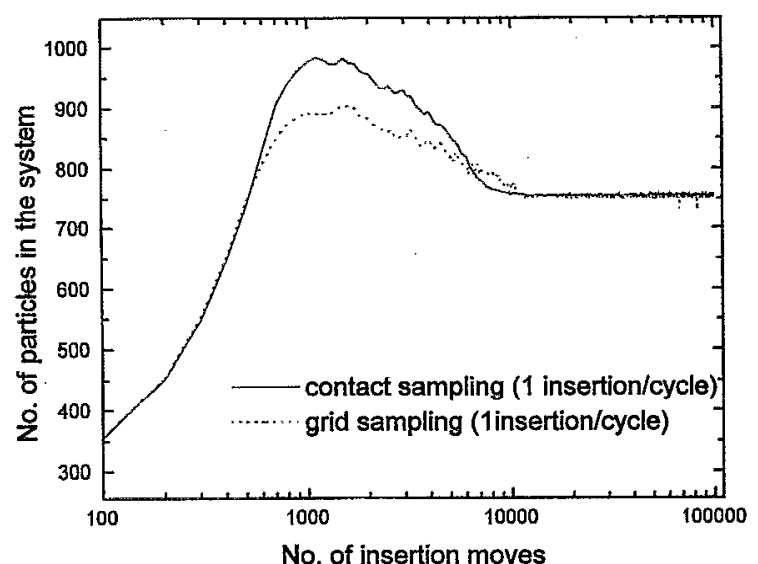

FIG. 6. Approach to equilibrium at $z^{\prime}=100$ for the two cavity-biased methods.

contact cavity biased algorithm. With more frequent insertion attempts per cycle, the contact biased method appears to converge much faster on the basis of total MC moves as shown in Fig. 8. The total number of MC moves is 100 times less than the fixed grid algorithm.

In Mezei's method, a finer grid is required at higher densities. ${ }^{7}$ It is reported that grids less than 0.2 sigma apart are required. In the runs of Figs. 7 and 8, the grids are about one sigma apart and could have led to the inefficiency and errors of the Mezei's method (especially at the final density). A simulation run with a finer grid is thus performed. The starting density is $\rho^{*}=0.7$ and the final density is about $\rho^{*}=0.95$. There are 28 grids on each side of the simulation box. To implement a fine grid, the cpu time required increases in proportion to the increased number of grid points. Hence, a smaller system of 120 particles at the starting density of $\rho^{*}=0.7$ is used. The grids are about 0.18 sigma apart at the final density. In comparison, a parallel run using new method was made with the same number of cavity test points. The number of test points for contact cavities in-

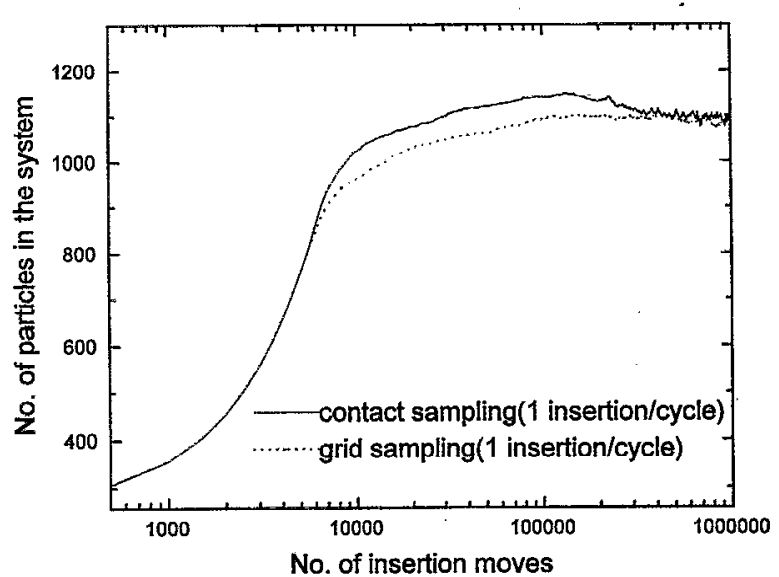

FIG. 7. Approach to equilibrium at $z^{\prime}=100000$ for the two cavity-biased methods.

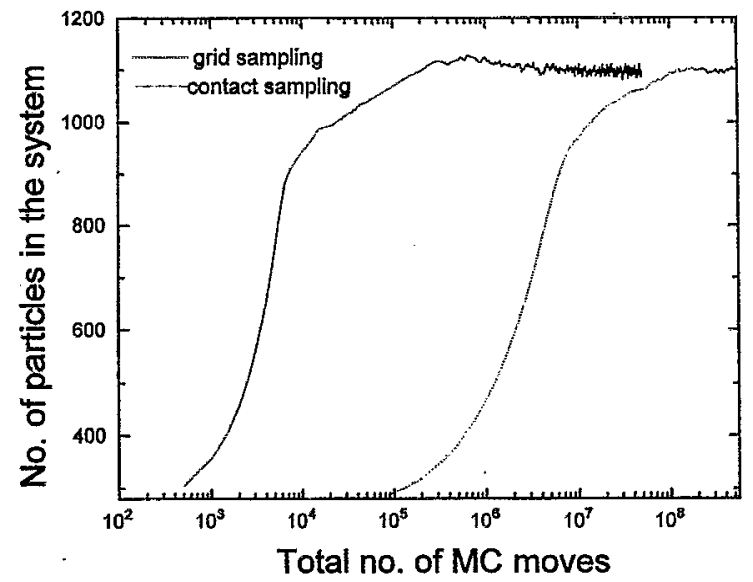

FIG. 8. Approach to equilibrium at $z^{\prime}=100000$ in terms of total MC moves.

creased to about 180 per particle. The results in Fig. 9 show a smaller disparity between the two methods in the onset of density increase probably due to a higher starting density. The slope of density increase of the contact sampling method seems to be steeper and it outperforms the grid method in Figs. 8 and 9 . The grid method converges slower and have a lower final density of $\rho^{*}=0.928$ compared to $\rho^{*}=0.944$ in the contact sampling method. The corresponding cpu time is plotted in Fig. 10 and it shows that the grid method is about 10 times slower in reaching convergence.

Further test runs are made for a range of excess chemical potential. The link cell ${ }^{13}$ method is used to reduce the cpu time by 3 to 4 times. In these runs, 1000 test points are used for cavities sampling and the starting configuration is $N=256$ at $\rho^{*}=0.2$ for all the runs. More insertions are made in each cycle in the contact cavity biased method. The results are tabulated in Table $\Pi$ and plotted in Fig. 11. The reduced activity is the input variable in the simulation but is converted to excess chemical potential with $\beta \mu^{\mathrm{ex}}=\ln \left(z^{\prime} / \rho^{*}\right)$ in Fig. 11. The contact insertion method requires about the

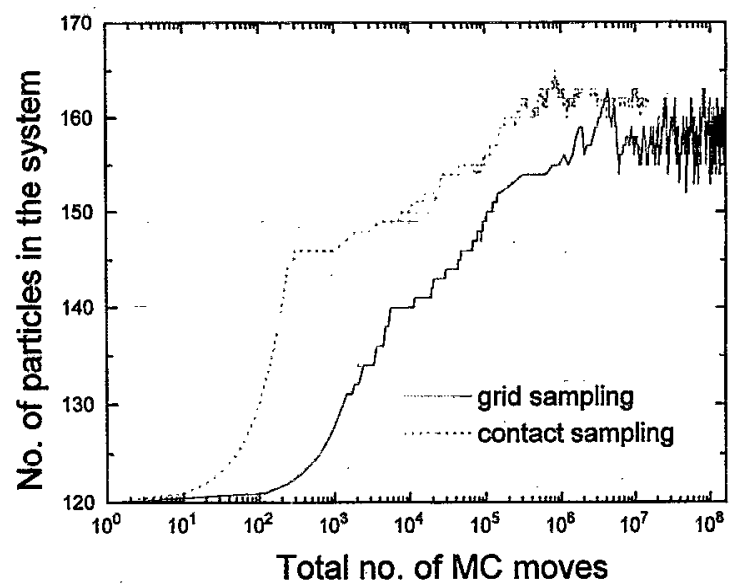

FIG. 9. Approach to equilibrium for a run with grid lines $0.18 \sigma$ apart and 180 test points per particle at $z^{\prime}=1 e 7$. 


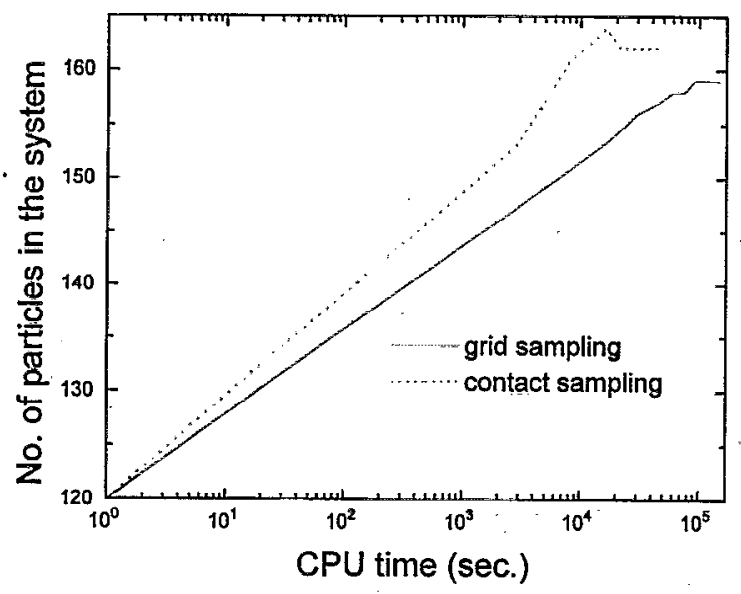

FIG. 10. cpu time from starting configuration to equilibrium for the two cavity-biased methods at $z^{\prime}=1 e 7$.

same number of insertion attempts to converge but many less MC moves. Furthermore, at high excess chemical potential, the contact cavity biased scheme will converge to a density in agreement with the Carnahan and Starling equation of state. The grid method fails to reach a density higher than 0.9340 despite further increase of $\mu^{\mathrm{ex}}$. The contact method also fails to reach density higher than 0.9880 . At this density, it may be near or actually inside the solid-liquid transition region. ${ }^{14,15}$ From the distribution function obtained, however, the simulation runs have not reached the state with solid state structures.

The pressure in each run is calculated from the contact value of $g(r)$. The resulting compressibility factors are plotted in Fig. 12. The agreement with the Carnahan and Starling equation of state is good.

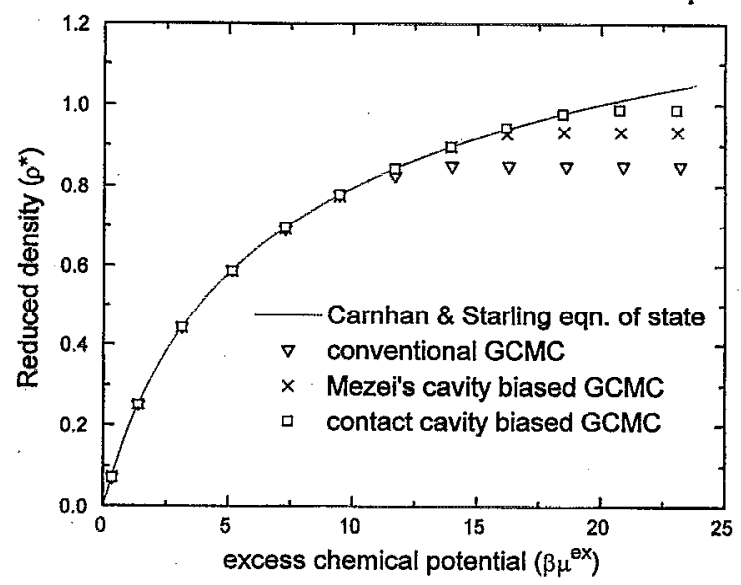

FIG. 11. The results of final reduced densities $\left(\rho^{*}\right)$ against excess chemical potential for the three GCMC methods and the Carnahan and Starling equation of state.

From the test runs of this work, the contact cavity biased algorithm is as accurate and efficient as the grid method at moderate densities. At high densities, it appears to be superior in efficiency and accuracy. In all the tests runs, both $g(\sigma)$ or $P_{c}(\sigma)$ are obtained during the simulation. If a prior knowledge of $g(\sigma)$ or $P_{c}(\infty)$ is assumed, e.g., using the VerletWeis algorithm ${ }^{16}$ or Eq. (11), much saving in cpu time can be achieved since cavity sampling can stop with the first cavity found. The new method and the grid method both suffer the potential problem of an approximate assumption in the microscopic reversibility of the insertion and deletion steps. The insertions are made into specific cavities but deletions can occur anywhere: This imperfectness, however, is less severe in the new method because the cavities positions are

TABLE II. Test runs to compare conventional GCMC, Mezei's cavity biased GCMC, and contact cavity biased GCMC methods, where $z^{\prime}=z \sigma^{3}$ and $z$ is the activity $\left[z=\exp (\beta \mu) / \Lambda^{3}, \beta \mu^{\operatorname{ex}}=\ln \left(z^{\prime} / \rho^{*}\right)\right]$, and final $\rho^{*}$ is the final reduced densities of the corresponding $z^{\prime}$. Insertion moves is the number of insert/delete attempts to reach the final $\rho^{*}$. Total MC moves is the number of insertion + displacement attempts to reach the final $\rho^{*}$. The starting configuration is $\rho^{*}=0.2$ with 256 particles.

\begin{tabular}{|c|c|c|c|c|c|c|c|c|c|}
\hline \multirow{2}{*}{$\begin{array}{l}\text { Reduced } \\
\text { activity } \\
z^{\prime}\end{array}$} & \multicolumn{3}{|c|}{$\begin{array}{c}\text { Final } \\
\rho^{*}\end{array}$} & \multicolumn{3}{|c|}{$\begin{array}{l}\text { Insertion } \\
\text { moves }\end{array}$} & \multicolumn{3}{|c|}{$\begin{array}{l}\text { Total } \\
\text { MC } \\
\text { moves }\end{array}$} \\
\hline & a & $b$ & c & a & b & $\mathrm{c}$ & a & b & $\mathrm{c}$ \\
\hline 0.1 & 0.071 & 0.071 & 0.071 & $1 \times 10^{5}$ & $1 \times 10^{4}$ & $1 \times 10^{4}$ & $1 \times 10^{5}$ & $1.6 \times 10^{6}$ & $1 \times 10^{5}$ \\
\hline 1 & 0.2516 & 0.2518 & 0.2509 & $1 \times 10^{5}$ & $1 \times 10^{4}$ & $1 \times 10^{4}$ & $1 \times 10^{5}$ & $2.9 \times 10^{6}$ & $1 \times 10^{5}$ \\
\hline 10 & 0.4438 & 0.4435 & 0.4430 & $1 \times 10^{5}$ & $1 \times 10^{4}$ & $1 \times 10^{4}$ & $1 \times 10^{5}$ & $8.6 \times 10^{6}$ & $1 \times 10^{5}$ \\
\hline 100 & 0.5884 & 0.5886 & 0.5860 & $1 \times 10^{5}$ & $1 \times 10^{4}$ & $1 \times 10^{4}$ & $1 \times 10^{5}$ & $1.1 \times 10^{7}$ & $1 \times 10^{5}$ \\
\hline 1000 & 0.6914 & 0.6922 & 0.6959 & $1 \times 10^{5}$ & $1 \times 10^{4}$ & $1 \times 10^{4}$ & $1 \times 10^{5}$ & $1.4 \times 10^{7}$ & $1 \times 10^{5}$ \\
\hline $1 e 4$ & 0.7767 & 0.7742 & 0.7780 & $2 \times 10^{5}$ & $1 \times 10^{4}$ & $1 \times 10^{4}$ & $2 \times 10^{5}$ & $1.8 \times 10^{7}$ & $1 \times 10^{5}$ \\
\hline Ies & 0.8252 & 0.8407 & 0.8422 & $1.2 \times 10^{7}$ & $3 \times 10^{5}$ & $3 \times 10^{5}$ & $1.2 \times 10^{7}$ & $3.1 \times 10^{8}$ & $3 \times 10^{6}$ \\
\hline $1 e 6$ & 0.8500 & 0.8964 & 0.8964 & $1.2 \times 10^{7}$ & $4 \times 10^{5}$ & $4 \times 10^{5}$ & $1.2 \times 10^{7}$ & $4.1 \times 10^{8}$ & $4 \times 10^{6}$ \\
\hline $1 e 7$ & 0.8500 & 0.9289 & 0.9440 & $1.2 \times 10^{7}$ & $5 \times 10^{6}$ & $5 \times 10^{6}$ & $1.2 \times 10^{7}$ & $5.5 \times 10^{9}$ & $5 \times 10^{7}$ \\
\hline $1 e 8$ & 0.8500 & 0.9340 & 0.9773 & $1.2 \times 10^{7}$ & $6 \times 10^{6}$ & $6 \times 10^{6}$ & $1.2 \times 10^{7}$ & $6.7 \times 10^{9}$ & $6 \times 10^{7}$ \\
\hline $1 e 9$ & 0.8500 & 0.9340 & 0.9880 & $1.2 \times 10^{7}$ & $6 \times 10^{6}$ & $6 \times 10^{6}$ & $1.2 \times 10^{7}$ & $6.7 \times 10^{9}$ & $6 \times 10^{7}$ \\
\hline $1 e 10$ & 0.8500 & 0.9340 & 0.9880 & $1.2 \times 10^{7}$ & $6 \times 10^{6}$ & $6 \times 10^{6}$ & $1.2 \times 10^{7}$ & $6.7 \times 10^{9}$ & $6 \times 10^{7}$ \\
\hline
\end{tabular}

a Results of conventional GCMC method.

hesults of the Mezei's cavity biased GCMC method with 1000 cavity test points.

${ }^{c}$ Results of the contact cavity biased GCMC method with insertion position at 1.0001 sigma for 1000 cavity test points. 


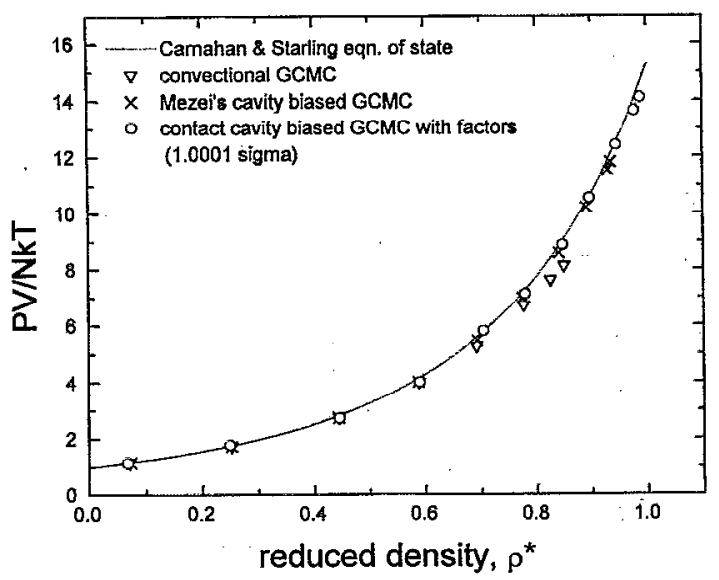

FIG. 12. Comparison of compressibility factors $(P V / N k T)$ against reduced densities $\left(\rho^{*}\right)$ for the three GCMC methods and the Carnahan and Starling equation of state.

relative to particles' coordinates which will change throughout the simulation and eventually cover the entire space. The cavities will be uniformly distributed given adequate statistics. In the grid method, the cavities are on a prefixed grid and not uniformly distributed over the coordinate space of the simulation box. It may also be attractive to test the contact cavity bias further for mixtures of hard spheres and in inhomogeneous systems. For systems with other intermolecular potentials, correlation of cavities may not follow the radial distribution functions. Modification of the contact cavity biased algorithm for these systems will be necessary. While we notice the contact peak of pair distribution can be high at high densities. It will be even higher for a contact triplet. Insertion at positions contacting existing contact pairs may have a very high probability of success. An algorithm taking advantage of this may have even higher efficiency.

\section{CONCLUSIONS}

Modifications of the cavity sampling method in the cavity-biased GCMC simulation can lead to possible improvements. Abandoning a fixed grid of cavity test points allow more frequent sampling of cavities between displacement cycles. By taking advantage of the radial correlation of cavities, insertion at positions contacting existing particles will be easier to succeed. Comparisons of these variations of GCMC methods show the contact cavity-biased scheme to be slightly advantageous and is open to further improvements.

\section{ACKNOWLEDGMENTS}

This work has been funded by the Committee on $\mathrm{Re}$ search and Conference Grants (CRGC) of the University of Hong Kong. Financial support to Danny H. L. Yau by the Croucher Foundation and the Hsin Chong-K. N. Godfrey Yeh Education Fund is acknowledged.

${ }^{1}$ D. J. Adams, Mol. Phys. 28, 1241 (1974).

${ }^{2}$ G. E. Norman and V. S. Filinov, High Temp. (USSR) 7, 216 (1969).

${ }^{3}$ L. A. Rowley, D. Nicholson, and N. G. Parsonage, J. Comput. Phys. 46, 1109 (1982).

${ }^{4}$ M. Mezei, Mol. Phys. 40, 901 (1980).

${ }^{5}$ K. E. Gubbins, K. S. Shing, and W. B. Streett, J. Phys. Chem. 87, 4579 (1983).

${ }^{6}$ M. Mezei, Molecular Simulations 9, 257 (1992).

${ }^{7}$ M. Mezei, Mol. Phys. 61, 565 (1987); 67, 1207(E) (1989).

${ }^{8}$ H. Reiss, H. L. Frisch, and J. L. Lebowitz, J. Chem. Phys. 31, 369 (1959).

${ }^{9}$ N. F. Carnahan and K. E. Starling, J. Chem. Phys, 51, 635 (1969).

${ }^{10}$ B. Widom, J. Chem. Phys. 39, 2808 (1963).

${ }^{11}$ B. Widom, J. Phys. Chem. 86, 869 (1982).

${ }^{12}$ Luis E. S. de Souza, Argyroula Stamatopoulou, and Dor Ben-Amotz, J. Chem. Phys. 100, 1456 (1994).

${ }^{13}$ B. Quentrec and C. Brot, J. Comput. Phys. 13, 430 (1975).

${ }^{14}$ W. G. Hoover and F. H. Ree, J. Chem. Phys. 49, 3609 (1968).

${ }^{15}$ B. J. Alder, W. G. Hoover, and D. A. Young, J. Chem. Phys. 49, 3688 (1968).

${ }^{16}$ L. Verlet and J. J. Weis, Phys. Rev. A 5, 939 (1972). 